\title{
Editorial
}

\section{Managing interaction, location, and communication in mobile information systems}

New paradigms like pervasive and mobile computing clearly show a trend of using computing and communication power to overcome the physical limitations posed by state-of-the-art information systems. Technologies like wireless networks, ambient intelligence, mobile agents, miniaturized computing devices, embedded systems, etc., have enabled a plethora of new types of applications and services. However, usage statistics show that there is still a huge gap between the potential of such services and the existing and newly developed approaches for the analysis, design, implementation and evaluation of mobile information systems.

This special issue of the International Journal of Mobile Information Systems (IJMIS) on "Managing Interaction, Location, and Communication in Mobile Information Systems" consists of four papers addressing some of the technical issues and constraints, interaction, location and communication have on the design and functionalities of mobile information systems .

The first paper Personalized Avatars for Mobile Entertainment by Tomislav Kosutic, Mario Weber, Miran Mosmondor, Ivan Andrisek, Maja Matijasevic, and Igor Pandzic describes a service, called LiveMail that uses existing face animation technologies for mobile entertainment. This prototype service allows mobile subscribers to communicate using personalized 3D face models created from images taken by phone cameras. These images are manipulated to create a 3D model of that face that can be animated simply by typing in some text. Speech and appropriate animation of the face are created automatically by speech synthesis. These personalized animations can be sent to others as real 3D animated messages or as short videos in MMS. The clients were implemented on different platforms, and different network and face animation techniques, and connected into one complex system.

The second paper A Generic Framework For Location-Based Services (LBS) Provisioning by Ioannis Priggouris, Stathes Hadjiefthymiades, Manos Spanoudakis, and Dimitrios Spanoudakis presents an integrated platform for delivering Location Based Services (LBS). The platform covers the full life cycle of LBS from the specification, deployment and maintenance of services, the service invocation and the final delivery of the produced results to the invoking user. A prototype implementation of the discussed platform was developed and used to perform a series of trial services, with the purpose of demonstrating the pursued functionality.

The third paper Location Based Mobile Computing - A Tuplespace Perspective by Anders Fongen , and Christian Larsen proposes a middleware for location and context management built on top of Mobispace. Mobispace is a distributed tuplespace made for mobile units (J2ME) where replication between local replicas takes place with a central server (over GPRS) or with other mobile units (using Bluetooth). Since a Bluetooth connection indicates physical proximity to another node, a set of stationary 
nodes may distribute locality information over Bluetooth connections, and this information may be retrieved through the ordinary tuplespace API.

The fourth paper Bluetooth: Opening a Blue Sky for Healthcare by X. H. Wang, M. Iqbal and R.S.H. Istepanian discusses the application of Bluetooth technology in healthcare. It describes the history of Bluetooth technology, its technical characteristics, and latest development. The applications are based on two basic types of links of Bluetooth technology: point-to-point link and point-to-multipoint link. The special requirements from healthcare and the challenges of successfully application of Bluetooth in healthcare are discussed.

Taking this opportunity, I would like to thank all the authors, referees, and journal editor-in-chief and editorial board for their contributions and valuable comments on this special issue.

Ismail Khalil Ibrahim

Guest Editor

Ismail Khalil Ibrahim is senior researcher and lecturer at the institute of Telecooperation at Johannes Kepler University Linz, Austria. He currently teaches, consults, and conducts research in Mobile Multimedia, Norm-Based Computing, and Ubiquitous Intelligence and is also interested in the broader business, social, and policy implications associated with the emerging Information Technologies. Dr. Ibrahim holds a B.Sc. in Electrical Engineering (1985), M.Sc., (1998) and Ph.D. (2001) in Computer Engineering and Information Systems. Before joining Johannes Kepler University Linz in October 2002, he was a research fellow at the Intelligent Systems Group at Utrecht University, Netherlands from 2001-2002 and a senior researcher and project manager at the Software Competence Center Hagenberg - Austria from 2000-2001.

Dr. Ibrahim is the founder and the steering committee chair of the international (iiWAS) series of conferences on Information Integration and Web-based Applications \& Services since 1999 and (MoMM) series of conferences on Advances in Mobile Multimedia since 2003, overseas Collaborator in the E-Commerce Lab at the National University of Singapore and the Telematics Lab at Bandung Institute of Technology, Indonesia. He serves as the editor-in-chief of the International Journal of Web Information Systems, Journal of Mobile Multimedia, editorial board of several journals, including, Business Process Management Journal, The International Journal of Information Technology and Web Engineering, Journal of Ubiquitous Computing and Intelligence, International Journal of Wireless and Mobile Computing, and the International Journal of Business Data Communications and Networking.

His work has been published at various books, journals and conferences. He is also reviewer and PC member for several conferences and journals. 

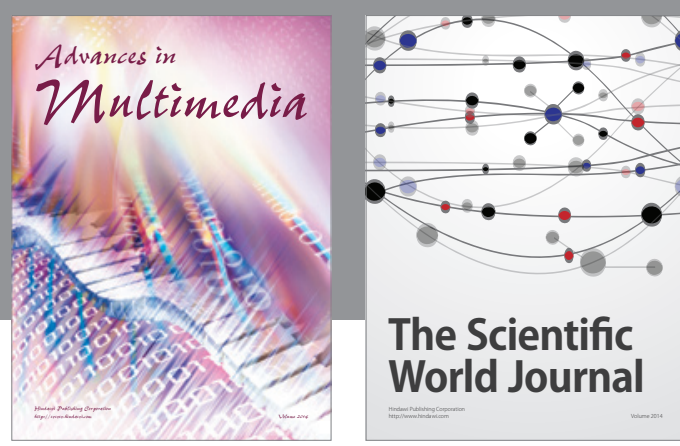

The Scientific World Journal
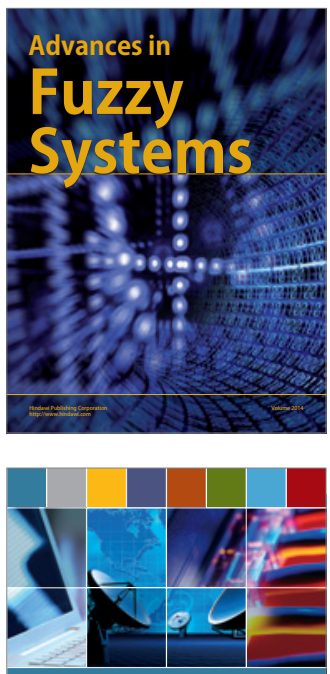

Computer Networks and Communications
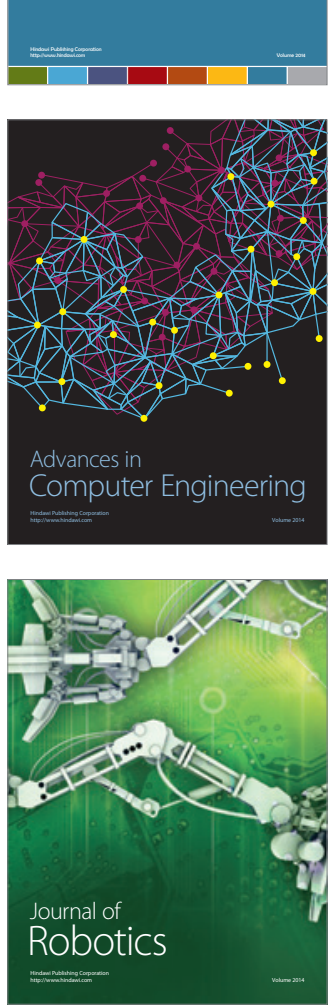
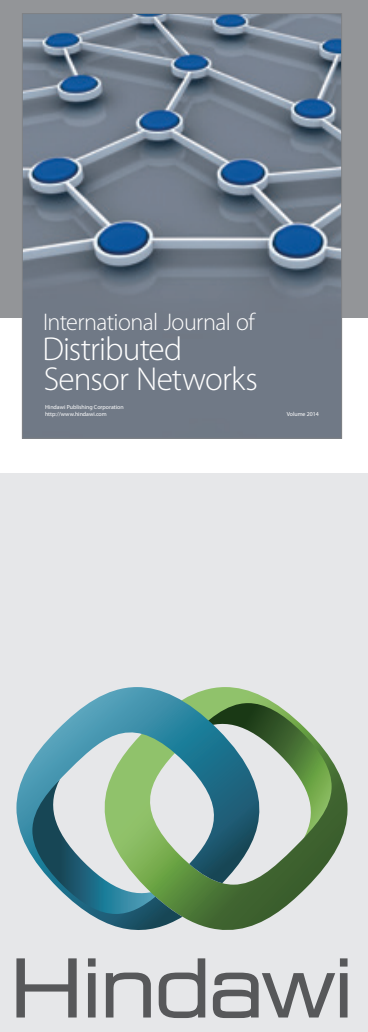

Submit your manuscripts at

http://www.hindawi.com
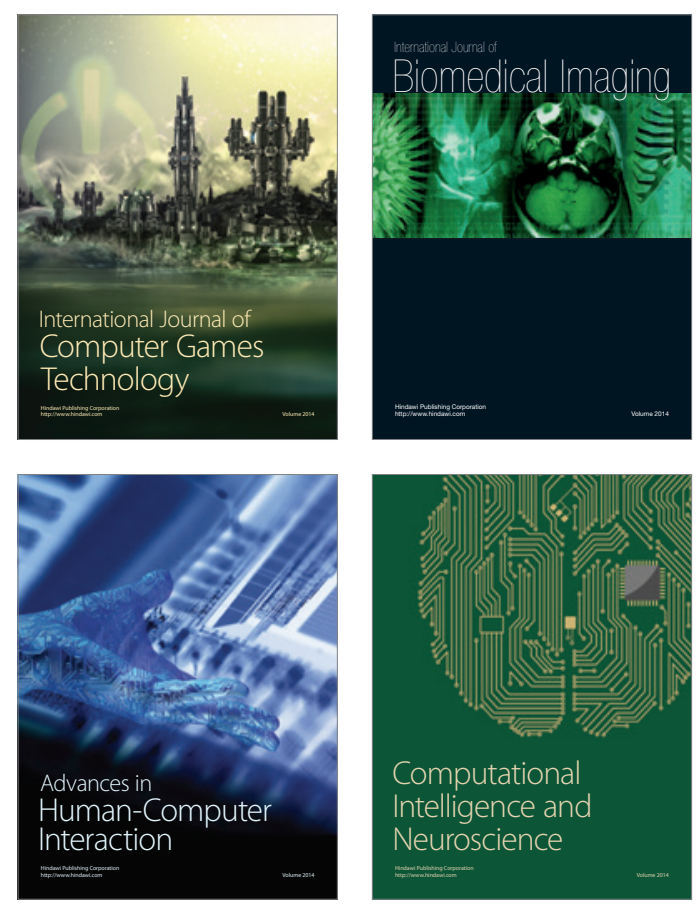
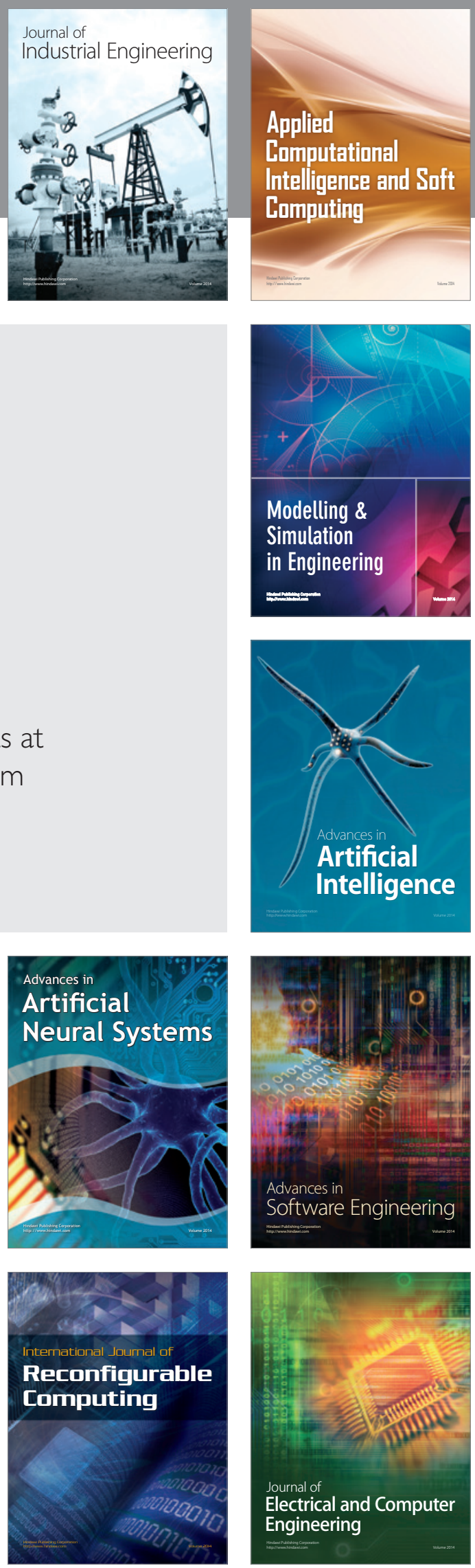